\title{
Robust consensus control of uncertain multi-agent systems with input delay: a model reduction method
}

DOI:

$10.1002 /$ rnc.3642

\section{Document Version}

Accepted author manuscript

Link to publication record in Manchester Research Explorer

\section{Citation for published version (APA):}

Zuo, Z., Wang, C., \& Ding, Z. (2017). Robust consensus control of uncertain multi-agent systems with input delay: a model reduction method. International Journal of Robust and Nonlinear Control, 27(11), 1874-1894. [RNC3642]. https://doi.org/10.1002/rnc.3642

\section{Published in:}

International Journal of Robust and Nonlinear Control

\section{Citing this paper}

Please note that where the full-text provided on Manchester Research Explorer is the Author Accepted Manuscript or Proof version this may differ from the final Published version. If citing, it is advised that you check and use the publisher's definitive version.

\section{General rights}

Copyright and moral rights for the publications made accessible in the Research Explorer are retained by the authors and/or other copyright owners and it is a condition of accessing publications that users recognise and abide by the legal requirements associated with these rights.

\section{Takedown policy}

If you believe that this document breaches copyright please refer to the University of Manchester's Takedown Procedures [http://man.ac.uk/04Y6Bo] or contact uml.scholarlycommunications@manchester.ac.uk providing relevant details, so we can investigate your claim.

\section{OPEN ACCESS}




\title{
Robust consensus control of uncertain multi-agent systems with input delay: a model reduction method ${ }^{\dagger}$
}

\author{
Zongyu Zuo ${ }^{1}$, Chunyan Wang ${ }^{2}$ and Zhengtao Ding ${ }^{2 *}$ \\ ${ }^{1}$ The Seventh Research Division, and Science and Technology on Aircraft Control Laboratory, Beihang University \\ (BUAA), Beijing 100191, P. R. China \\ ${ }^{2}$ Control Systems Centre, School of Electrical and Electronic Engineering, University of Manchester, Sackville Street \\ Building, Manchester M13 9PL, UK
}

\begin{abstract}
SUMMARY
This paper addresses the robust consensus control design for input-delayed multi-agent systems subject to parametric uncertainties. To deal with the input delay, the Artstein model reduction method is employed by a state transformation. The input-dependent integral term that remains in the transformed system, due to the model uncertainties, is judiciously analysed. By carefully exploring certain features of the Laplacian matrix, sufficient conditions for the global consensus under directed communication topology are identified using Lyapunov-Krasovskii functionals in the time domain. The proposed control only relies on relative state information of the subsystems via network connections. The effectiveness and robustness of the proposed control design is demonstrated through a numerical simulation example. Copyright (c) 2015 John Wiley \& Sons, Ltd.
\end{abstract}

Received ...

KEY WORDS: Consensus Control; Input Delay; Multi-agent Systems; Parametric Uncertainty; Reduction Method

\section{INTRODUCTION}

Control of network-connected systems, including formation control [1], flocking [2] and synchronization [3], has attracted significant interests in control area and has been intensively studied in the literature, [4]-[9], to mention a few. In some applications involving multi-agent systems, groups of agents are required to reach an agreement upon certain quantities of interest, which is called consensus or agreement problem. One significant advance in consensus control is to use tools in graph theory, especially the use of Laplacian matrices [10], to characterize the network connection.

Time delays are inevitable in industrial processes due to the time taken for transmission of signals. The importance of addressing delay has been well recognized for a long time (see [11] and the references therein). Time delay is even more significant in network-connected systems as the control inputs depend on the signals transmitted through network communication. The presence of communication delays, if not considered in a controller design, may seriously deteriorate the performance of the multi-agent networks. This problem has attracted the considerate attention in control community, as evidenced by recent publications [12]-[16]. In [17], it is pointed out that the delay in the network communication can also be viewed as input delay in consensus control .

\footnotetext{
${ }^{*}$ Correspondence to: Zhengtao Ding, Control Systems Centre, School of Electrical and Electronic Engineering, University of Manchester, Sackville Street Building, Manchester M13 9PL, UK. E-mail: zhengtao.ding@ manchester.ac.uk
} 
The practical physical systems often suffer from uncertainties which may be caused by mutations in system parameters, modelling errors or some ignored factors [18]. The robust consensus problem of multi-agent systems has formed into a challenge topic in recent years. Han et al. investigate the robust consensus problem for multi-agent systems with continuous-time and discrete-time dynamics in [19] and [20], where the weighted adjacency matrix is a polynomial function of uncertain parameters. In particular, the $H_{\infty}$ robust control problem is investigated in [21] for a group of autonomous agents governed by uncertain general linear node dynamics. However, most of the existing results on consensus control of uncertain multi-agent systems were often restricted to certain conditions, like single or double integrators [22], undirected network connections [23] or systems without delays [24]. The difficulty of solving the consensus problem with general uncertain model dynamics with time delay is mainly due to the fact that the systems can not be completely transformed to a delay-free one due to the existence of uncertainties. The nature of infinite-dimensionality of delay issues prevents the direct application of many well-know existing conventional control design tools. Further analysis is needed to tackle the influence of the extra integral terms under the transformations, including the ones for parametric uncertainty and input delay.

This paper systematically investigate the consensus control for general linear multi-agent systems with parametric uncertainties and communication delay. This kind of network communication delay can be formulated as the input delay when the inputs only depend on the relative state information transmitted via the network. A model reduction method, which was originally introduced by Artstein in [26], is used to deal with the input delay. The control design is only based on the neighbours' information obtained via the network connections, without local feedback of the subsystems. Further endeavors are made to ensure that the extra integral term, which remains in the system dynamics after transformation due to the parametric uncertainty and depends on the relative state due to the input, is properly considered. By transforming the Laplacian matrix into the real Jordan form, the consensus analysis is put in the framework of Lyapunov-Krasovskii functionals in real domain. A simulation example is included in the end of the paper.

The remainder of this paper is organised as follows. Some notations and the problem formulation are given in Section 2. Section 3 presents a couple of preliminary results for the stability analysis. Section 4 presents the main results on the consensus control design and stability analysis. Simulation results are included in Section 5. Section 6 concludes this paper.

\section{PROBLEM STATEMENT}

In this paper, we consider the control design for a set of $N$ uncertain subsystems with input delay, of which the subsystems are described by

$$
\dot{x}_{i}(t)=[A+\Delta A(t)] x_{i}(t)+[B+\Delta B(t)] u_{i}(t-h),
$$

where, for subsystem $i, i=1,2, \ldots, N, x_{i} \in \mathbb{R}^{n}$ is the state vector, $u_{i} \in \mathbb{R}^{m}$ is the control input vector, $A \in \mathbb{R}^{n \times n}$ and $B \in \mathbb{R}^{n \times m}$ are constant matrices with $(A, B)$ controllable, $h>0$ is the input delay, $x_{i}(\theta), \theta \in[-h, 0]$, are given and bounded, $\Delta A(t)$ and $\Delta B(t)$ are time-varying uncertain matrices which can be formulated in the form [27] as

$$
\Delta A(t)=E \Sigma(t) F_{1} \quad \text { and } \quad \Delta B(t)=E \Sigma(t) F_{2}
$$

where $E, F_{1}$ and $F_{2}$ are real constant matrices with appropriate dimensions, and $\Sigma(t)$ is an unknown real time-varying matrix that satisfies $\Sigma^{\mathrm{T}}(t) \Sigma(t) \leq I$.

\section{Remark 1}

It is worth noticing that the subsystems in the network are nominally identical and the model uncertainty matrices satisfy the same form as (2). Different from the existing works that focus on the identical agents in the network, the terms $\Delta A$ and $\Delta B$ in (1) allow the subsystems to have different dynamics and the uncertainty is characterised by the time-varying matrix $\Sigma(t)$, which implies that 
each subsystem in the group can be non-identical. For the consensus design, only the bound of $\Sigma(t)$ (i.e., the worst case) is needed.

\section{Assumption 1}

All subsystems in the network have known and identical input delays.

\section{Remark 2}

Assumption 1 is adopted for the convenience of illustration of the control design. The proposed method in this paper may be extended to the network-connected systems with non-identical constant delays and even unknown time-varying delays if the worst case is taken into account in the design.

The information flow among the subsystems is specified by a directed graph $\mathcal{G}$, which consists of a set of vertices denoted by $\mathcal{V}$ and a set of edges denoted by $\mathcal{E}$. A vertex represents a subsystem, and each edge represents a connection. Associated with the graph is its adjacency matrix $Q$, where element $q_{i j}$ denotes the connection among the subsystems. More specifically, $q_{i j}=1$ if there is a connection from subsystem $j$ to subsystem $i$, and $q_{i j}=0$ otherwise. The Laplacian matrix $L=\left\{l_{i j}\right\}$ is commonly defined by

$$
\begin{aligned}
& l_{i j}=-q_{i j}, \text { if } j \neq i, \\
& l_{i i}=\sum_{j=1, j \neq i}^{N} q_{i j} .
\end{aligned}
$$

From the definition of the Laplacian matrix, it is clear that

$$
L 1=0,
$$

where $\mathbf{1}=[1, \cdots, 1]^{\mathrm{T}} \in \mathbb{R}^{N}$, which implies that the Laplacian matrix has 0 as an eigenvalue associated with the right eigenvector 1 .

\section{Assumption 2}

0 is a simple eigenvalue of the Laplacian matrix.

In terms of the network collections, this condition implies that the network has a spanning tree to connect any two subsystems in the system. For consensus design, we only need that the eigenvalue at 0 is a single eigenvalue [28].

The consensus control problem considered in this paper is to design a control strategy, using the relative state information, to ensure that all input-delayed uncertain subsystems converge to an identical trajectory.

\section{PRELIMINARY RESULTS}

In this section, we present a couple of preliminary results which are useful for the stability analysis. We first present an overview of the Artstein model reduction method [25, 26]. Consider an inputdelayed system

$$
\dot{x}(t)=A x(t)+B u(t-h) .
$$

with $x(\theta), \theta \in[-h, 0]$, being given and bounded.

Introducing a new variable

$$
z(t)=x(t)+\int_{t}^{t+h} \mathrm{e}^{A(t-\tau)} B u(\tau-h) \mathrm{d} \tau
$$


reduces (3) to a delay-free system

$$
\begin{aligned}
\dot{z}(t) & =\dot{x}(t)+A \int_{t}^{t+h} \mathrm{e}^{A(t-\tau)} B u(\tau-h) \mathrm{d} \tau+e^{-A h} B u(t)-B u(t-h) \\
& =A x(t)+A \int_{t}^{t+h} \mathrm{e}^{A(t-\tau)} B u(\tau-h) \mathrm{d} \tau+e^{-A h} B u(t) \\
& =A z(t)+D u(t),
\end{aligned}
$$

where $D \triangleq e^{-A h} B$. We consider a controller

$$
u(t)=K z(t) .
$$

If the controller (6) stabilises the transformed system (5), then the original system (3) is also stable with the same controller.

\section{Remark 3}

The transformation from $x$ to $z$ exists initially, which is guaranteed by the specified boundedness of the initial state $x(\theta), \theta \in[-h, 0]$. The existence of the transformation at subsequent time instances depends on the integrability of $u$, which is then guaranteed by the boundedness of $z$, as established in the stability analysis.

To reveal the block diagonal structure of the transformed Laplacian matrix for stability analysis, we next recall a lemma from [28].

\section{Lemma 1}

For a Laplacian matrix that satisfies Assumption 2, there exits a similarity transformation $T$, with its first column being $T_{(1)}=\mathbf{1}$, such that

$$
T^{-1} L T=J,
$$

with $J$ being a block diagonal matrix in the real Jordan form

$$
J=\left[\begin{array}{ccccccc}
0 & & & & & & \\
& J_{2} & & & & & \\
& & \ddots & & & & \\
& & J_{p} & & & \\
& & & J_{p+1} & & \\
& & & & & \ddots & \\
& & & & & J_{q}
\end{array}\right]_{N \times N}
$$

where $J_{k} \in \mathbb{R}^{n_{k} \times n_{k}}, k=2,3, \cdots, p$, are the Jordan blocks for real eigenvalues $\lambda_{k}>0$ with the multiplicity $n_{k}$ in the form

$$
J_{k}=\left[\begin{array}{ccccc}
\lambda_{k} & 1 & & & \\
& \lambda_{k} & 1 & & \\
& & \ddots & \ddots & \\
& & & \lambda_{k} & 1 \\
& & & & \lambda_{k}
\end{array}\right]_{n_{k} \times n_{k}}
$$

and $J_{k} \in \mathbb{R}^{2 n_{k} \times 2 n_{k}}, k=p+1, p+2, \ldots, q$, are the Jordan blocks for conjugate eigenvalues $\alpha_{k} \pm$ $j \beta_{k}, \alpha_{k}>0$ and $\beta_{k}>0$, with the multiplicity $n_{k}$ in the form

$$
J_{k}=\left[\begin{array}{ccccc}
\nu\left(\alpha_{k}, \beta_{k}\right) & I_{2} & & & \\
& \nu\left(\alpha_{k}, \beta_{k}\right) & I_{2} & & \\
& & \ddots & \ddots & \\
& & & \nu\left(\alpha_{k}, \beta_{k}\right) & I_{2} \\
& & & & \nu\left(\alpha_{k}, \beta_{k}\right)
\end{array}\right]_{2 n_{k} \times 2 n_{k}}
$$


with $I_{2}$ the identity matrix in $\mathbb{R}^{2 \times 2}$ and

$$
\nu\left(\alpha_{k}, \beta_{k}\right)=\left[\begin{array}{cc}
\alpha_{i} & \beta_{i} \\
-\beta_{i} & \alpha_{i}
\end{array}\right]_{2 \times 2}
$$

We also need the following lemma from [29].

\section{Lemma 2}

For a positive definite matrix $P$, and a function $x:[a, b] \rightarrow \mathbb{R}^{n}$, with $a, b \in \mathbb{R}$ and $b>a$, the following inequality holds

$$
\left(\int_{a}^{b} x^{\mathrm{T}}(\tau) \mathrm{d} \tau\right) P\left(\int_{a}^{b} x(\tau) \mathrm{d} \tau\right) \leq(b-a) \int_{a}^{b} x^{\mathrm{T}}(\tau) P x(\tau) \mathrm{d} \tau .
$$

\section{CONSENSUS CONTROL}

For the multi-agent systems (1), we consider the linear transformation (4) by the reduction method. The original subsystems are transformed to

$$
\dot{z}_{i}(t)=(A+\Delta A) z_{i}(t)+D u_{i}(t)+\Delta B u_{i}(t-h)-\Delta A \int_{t}^{t+h} \mathrm{e}^{A(t-\tau)} B u_{i}(\tau-h) \mathrm{d} \tau,
$$

where $D=e^{-A h} B$. As seen in (10), system (1) is not completely reduced to a free-of-delay system due to the model uncertainties.

We propose a control design using the relative state information. The control input takes the structure

$$
u_{i}(t)=-K \sum_{j=1}^{N} q_{i j}\left[z_{i}(t)-z_{j}(t)\right]=-K \sum_{j=1}^{N} l_{i j} z_{j}(t),
$$

where $K \in \mathbb{R}^{m \times n}$ is a constant control gain matrix to be designed later.

\section{Remark 4}

It is worth noting from (4) that the proposed control in (11) only uses the relative state information of the subsystems via network connections.

\section{Remark 5}

Note that the information on each control input $u_{i}(t)$ on the time interval $[t-h, t]$ can be stored and used for control. In practical implementations, the discretization of an integral or some numerical quadrature method [31] can be used to approximate the integral term in the control input $u_{i}(t)$.

\section{Remark 6}

For unknown non-identical time-varying delays $h_{i}(t)$ in (1) satisfying $0<h_{i}(t)<\bar{h}, i=$ $1,2, \ldots, N$, the upper bound of the delays that exist in all agents can be used in the predictor variable (4) as

$$
z_{i}(t)=x_{i}(t)+\int_{t}^{t+\bar{h}} e^{A(t-\tau)} B u_{i}(\tau-\bar{h}) \mathrm{d} \tau
$$

Then, the original subsystems (1) can be transformed to

$$
\begin{aligned}
\dot{z}_{i}(t)= & (A+\triangle A) z_{i}(t)+e^{-A \bar{h}} B u_{i}(t)-\triangle A \int_{t}^{t+\bar{h}} e^{A(t-\tau)} B u_{i}(\tau-\bar{h}) \mathrm{d} \tau+\Delta B u_{i}\left(t-h_{i}(t)\right) \\
& +B\left(u_{i}\left(t-h_{i}(t)\right)-u_{i}(t-\bar{h})\right) \\
= & (A+\triangle A) z_{i}(t)+\bar{D} u_{i}(t)-\triangle A \int_{t}^{t+\bar{h}} e^{A(t-\tau)} B u_{i}(\tau-\bar{h}) \mathrm{d} \tau+\triangle B u_{i}(t-\bar{h}) \\
& -B K \sum_{j=1}^{N} l_{i j} \int_{t-\bar{h}}^{t-h_{i}(t)} \dot{z}_{j}(\tau) \mathrm{d} \tau-\triangle B K \sum_{j=1}^{N} l_{i j} \int_{t-\bar{h}}^{t-h_{i}(t)} \dot{z}_{j}(\tau) \mathrm{d} \tau,
\end{aligned}
$$


where $\bar{D}=e^{-A \bar{h}} B$. Compared with (10), the use of the upper-bound of delays leads to additional integral terms in (13), which further complicates the design. Fortunately, with the same control structure (11), our proposed design method is still applicable if $K$ is carefully identified by introducing additional Krasovskii functionals in consensus analysis. Similar technique can be found in [32] for a single system with unknown time-varying delay but without parametric uncertainties.

Let $z(t)=\left[z_{1}, z_{2}, \ldots, z_{N}\right]^{\mathrm{T}}$, and the closed-loop system is then written as

$$
\dot{z}(t)=\left[I_{N} \otimes(A+\Delta A)-L \otimes D K\right] z(t)-(L \otimes \Delta B K) z(t-h)-\left(I_{N} \otimes \Delta A\right) \sigma(t),
$$

where $\sigma \triangleq\left[\sigma_{1}, \ldots, \sigma_{N}\right]^{\mathrm{T}}$ with the elements defined by

$$
\sigma_{i}=-\int_{t}^{t+h} \mathrm{e}^{A(t-\tau)} B K \sum_{j=1}^{N} l_{i j} z_{j}(\tau-h) \mathrm{d} \tau,
$$

and $\otimes$ denotes the Kronecker product of matrices.

Let us define $r \in \mathbb{R}^{N}$ as the left eigenvector of $L$ corresponding to the eigenvalue at 0 , that is, $r^{\mathrm{T}} L=0$. Furthermore, let $r$ be scaled such that $r^{\mathrm{T}} \mathbf{1}=1$. It can be shown from Assumption 2 and Lemma 1 that there exists a non-singular matrix $T$ with its first column being $T_{(1)}=\mathbf{1}$ and the first row of $T^{-1}$ being $T_{(1)}^{-1}=r^{\mathrm{T}}$, such that

$$
T^{-1} L T=J .
$$

Based on the vector $r$, we introduce a state transformation

$$
\xi_{i}(t)=z_{i}(t)-\sum_{j=1}^{N} r_{j} z_{j}(t)
$$

for $i=1, \cdots, N$. With

$$
\xi(t)=\left[\xi_{1}^{\mathrm{T}}(t), \xi_{2}^{\mathrm{T}}(t), \ldots, \xi_{N}^{\mathrm{T}}(t)\right]^{\mathrm{T}},
$$

we have

$$
\xi(t)=z(t)-\left[\left(\mathbf{1} r^{\mathrm{T}}\right) \otimes I_{n}\right] z(t)=\left(M \otimes I_{n}\right) z(t),
$$

where $M \triangleq I_{N}-\mathbf{1} r^{\mathrm{T}}$. Since $r^{\mathrm{T}} \mathbf{1}=1$, it can be shown that $M \mathbf{1}=0$. Therefore, the consensus of system (14) is achieved when $\xi=0$, as $\xi=0$ implies $z_{1}=z_{2}=\cdots=z_{N}$, due to the fact the null space of $M$ is $\operatorname{span}\{\mathbf{1}\}$. Then, the consensus problem is now converted to the stabilization problem.

The dynamics of $\xi(t)$ can then be obtained as

$$
\begin{aligned}
\dot{\xi}(t) & =\left(M \otimes I_{n}\right) \dot{z}(t) \\
& =\left[I_{N} \otimes(A+\Delta A)-L \otimes D K\right] \xi(t)-(L \otimes \Delta B K) \xi(t-h)-\left(M \otimes I_{n}\right)\left(I_{N} \otimes \Delta A\right) \sigma .
\end{aligned}
$$

To explore the structure of $L$ for stability analysis, let us introduce another state transformation

$$
\eta(t)=\left(T^{-1} \otimes I_{n}\right) \xi(t)
$$

Then, we have

$$
\dot{\eta}(t)=\left[I_{N} \otimes(A+\Delta A)-J \otimes D K\right] \eta(t)-(J \otimes \Delta B K) \eta(t-h)-\Psi(z),
$$

where $\Psi(z)=\left(T^{-1} \otimes I_{n}\right)\left(M \otimes I_{n}\right)\left(I_{N} \otimes \Delta A\right) \sigma, \eta(t)=\left[\eta_{1}(t), \eta_{2}(t), \ldots, \eta_{N}(t)\right]^{\mathrm{T}}$ and $\Psi(z)=$ $\left[\psi_{1}(z), \psi_{2}(z), \ldots, \psi_{N}(z)\right]^{\mathrm{T}}$ with $\eta_{i} \in \mathbb{R}^{n}$ and $\psi_{i}: \mathbb{R}^{n N} \rightarrow \mathbb{R}^{n}$, for $i=1,2, \ldots, N$.

With the state transformations (17) and (19), we have

$$
\eta_{1}(t)=\left(r^{\mathrm{T}} \otimes I_{n}\right) \xi(t)=\left[\left(r^{\mathrm{T}} M\right) \otimes I_{n}\right] z(t) \equiv 0 .
$$


The consensus control can be guaranteed by showing that $\eta$ converges to zero, which is sufficed by showing that $\eta_{i}$ converge to zero for $k=2,3, \ldots, N$, since we have shown that $\eta_{1}(t) \equiv 0$.

With the control law shown in (11), we design the control gain matrix $K$ as

$$
K=D^{\mathrm{T}} P
$$

where $P$ is a positive definite matrix to satisfy certain condition. In the remaining part of the paper, we will use Lyapunov-function-based analysis to identify conditions for $P$ to ensure that the consensus control objective is achieved by using the control input (11) with the control gain (22).

The stability analysis will be carried out for $\eta$. Based on the structure of the Laplacian matrix shown in (8), we can see that

$$
N_{k}=n_{1}+\sum_{j=2}^{k} n_{j},
$$

with $n_{1}=1$ and $N_{q}=N$, where $k=2,3, \ldots, q$.

Due to the eigenstructure (8), the dynamics of the transformed state $\eta$ in (20) will be discussed corresponding to the real and the complex eigenvalues, respectively.

For the state variables associated with the Jordan blocks $J_{k}$ of real eigenvalues, i.e., for $2 \leq k \leq p$, we have the dynamics

$$
\begin{aligned}
\dot{\eta}_{i}(t)= & \left(A+\Delta A-\lambda_{i} D D^{\mathrm{T}} P\right) \eta_{i}(t)-D D^{\mathrm{T}} P \eta_{i+1}(t)-\lambda_{i} \Delta B D^{\mathrm{T}} P \eta_{i}(t-h) \\
& -\Delta B D^{\mathrm{T}} P \eta_{i+1}(t-h)-\psi_{i}(z), \quad i=N_{k-1}+1, N_{k-1}+2, \ldots, N_{k}-1, \\
\dot{\eta}_{i}(t)= & \left(A+\Delta A-\lambda_{i} D D^{\mathrm{T}} P\right) \eta_{i}(t)-\lambda_{i} \Delta B D^{\mathrm{T}} P \eta_{i}(t-h)-\psi_{i}(z), \quad i=N_{k} .
\end{aligned}
$$

For the state variables associated with the Jordan blocks $J_{k}$ of conjugate complex eigenvalues, i.e., for $k>p$, we consider the dynamics of the state variables in pairs. For notational convenience, let

$$
i_{1}(j)=N_{k-1}+2 j-1 \quad \text { and } \quad i_{2}(j)=N_{k-1}+2 j,
$$

where $j=1,2, \ldots, n_{k} / 2$. The dynamics of $\eta_{i_{1}(j)}$ and $\eta_{i_{2}(j)}$, for $j=1,2, \ldots, n_{k} / 2-1$, are expressed by

$$
\begin{aligned}
\dot{\eta}_{i_{1}(j)}(t)= & \left(A+\Delta A-\alpha_{k} D D^{\mathrm{T}} P\right) \eta_{i_{1}(j)}(t)-\beta_{k} D D^{\mathrm{T}} P \eta_{i_{2}(j)}(t)-D D^{\mathrm{T}} P \eta_{i_{1}(j)+2}(t) \\
& -\alpha_{k} \Delta B D^{\mathrm{T}} P \eta_{i_{1}(j)}(t-h)-\beta_{k} \Delta B D^{\mathrm{T}} P \eta_{i_{2}(j)}(t-h)-\Delta B D^{\mathrm{T}} P \eta_{i_{1}(j)+2}(t-h) \\
& -\psi_{i_{1}(j)}(z), \\
\dot{\eta}_{i_{2}(j)}(t)= & \left(A+\Delta A-\alpha_{k} D D^{\mathrm{T}} P\right) \eta_{i_{2}(j)}(t)+\beta_{k} D D^{\mathrm{T}} P \eta_{i_{1}(j)}(t)-D D^{\mathrm{T}} P \eta_{i_{2}(j)+2}(t) \\
& -\alpha_{k} \Delta B D^{\mathrm{T}} P \eta_{i_{2}(j)}(t-h)+\beta_{k} \Delta B D^{\mathrm{T}} P \eta_{i_{1}(j)}(t-h)-\Delta B D^{\mathrm{T}} P \eta_{i_{2}(j)+2}(t-h) \\
& -\psi_{i_{2}(j)}(z),
\end{aligned}
$$

and, for $j=n_{k} / 2$,

$$
\begin{aligned}
\dot{\eta}_{i_{1}(j)}= & \left(A+\Delta A-\alpha_{k} D D^{\mathrm{T}} P\right) \eta_{i_{1}(j)}(t)-\beta_{k} D D^{\mathrm{T}} P \eta_{i_{2}(j)}(t) \\
& -\alpha_{k} \Delta B D^{\mathrm{T}} P \eta_{i_{1}(j)}(t-h)-\beta_{k} \Delta B D^{\mathrm{T}} P \eta_{i_{2}(j)}(t-h)-\psi_{i_{1}(j)}(z), \\
\dot{\eta}_{i_{2}(j)}= & \left(A+\Delta A-\alpha_{k} D D^{\mathrm{T}} P\right) \eta_{i_{2}(j)}(t)+\beta_{k} D D^{\mathrm{T}} P \eta_{i_{1}(j)}(t) \\
& -\alpha_{k} \Delta B D^{\mathrm{T}} P \eta_{i_{2}(j)}(t-h)+\beta_{k} \Delta B D^{\mathrm{T}} P \eta_{i_{1}(j)}(t-h)-\psi_{i_{2}(j)}(z) .
\end{aligned}
$$

Let

$$
W_{i}=\eta_{i}^{\mathrm{T}}(t) P \eta_{i}(t)
$$


For $i=N_{k-1}+1, N_{k-1}+2, \ldots, N_{k}-1$, the time derivative of $W_{i}$ along the trajectory (24) is

$$
\begin{aligned}
\dot{W}_{i}= & \eta_{i}^{\mathrm{T}}(t) P \dot{\eta}_{i}(t) \\
= & \eta_{i}^{\mathrm{T}}(t)\left(A^{\mathrm{T}} P+P A-2 \lambda_{k} P D D^{\mathrm{T}} P\right) \eta_{i}(t)+2 \eta_{i}^{\mathrm{T}}(t) P \Delta A \eta_{i}(t)-2 \eta_{i}^{\mathrm{T}}(t) P D D^{T} P \eta_{i+1}(t) \\
& -2 \lambda_{k} \eta_{i}^{\mathrm{T}}(t) P \Delta B D^{\mathrm{T}} P \eta_{i}(t-h)-2 \eta_{i}^{\mathrm{T}}(t) P \Delta B D^{\mathrm{T}} P \eta_{i+1}(t-h)-2 \eta_{i}^{\mathrm{T}}(t) P \psi_{i} \\
\leq & \eta_{i}^{\mathrm{T}}(t)\left(A^{\mathrm{T}} P+P A-2 \lambda_{k} P D D^{\mathrm{T}} P\right) \eta_{i}(t)+\frac{1}{\mu} \eta_{i}^{\mathrm{T}}(t) P E E^{\mathrm{T}} P \eta_{i}(t)+\mu \eta_{i}^{\mathrm{T}}(t) F_{1}^{\mathrm{T}} F_{1} \eta_{i}(t) \\
& +\eta_{i}^{\mathrm{T}}(t) P D D^{\mathrm{T}} P \eta_{i}(t)+\eta_{i+1}^{\mathrm{T}}(t) P D D^{\mathrm{T}} P \eta_{i+1}(t)+\frac{1}{\epsilon} \lambda_{k} \eta_{i}^{\mathrm{T}}(t) P E E^{\mathrm{T}} P \eta_{i}(t) \\
& +\epsilon \lambda_{k} \eta_{i}^{\mathrm{T}}(t-h) P D F_{2}^{\mathrm{T}} F_{2} D^{\mathrm{T}} P \eta_{i}(t-h)+\frac{1}{\epsilon} \eta_{i}^{\mathrm{T}}(t) P E E^{\mathrm{T}} P \eta_{i}(t) \\
& +\epsilon \eta_{i+1}^{\mathrm{T}}(t-h) P D F_{2}^{\mathrm{T}} F_{2} D^{\mathrm{T}} P \eta_{i+1}(t-h)-2 \eta_{i}^{\mathrm{T}}(t) P \psi_{i} \\
= & \eta_{i}^{\mathrm{T}}(t)\left[A^{\mathrm{T}} P+P A-\left(2 \lambda_{k}-1\right) P D D^{\mathrm{T}} P+\left(\frac{\lambda_{k}+1}{\epsilon}+\frac{1}{\mu}\right) P E E^{\mathrm{T}} P+\mu F_{1}^{\mathrm{T}} F_{1}\right] \eta_{i}(t) \\
& +\eta_{i+1}^{\mathrm{T}}(t) P D D^{\mathrm{T}} P \eta_{i}(t)+\epsilon\left(1+\lambda_{k}\right) \eta_{i+1}^{T}(t-h) P D F_{2}^{\mathrm{T}} F_{2} D^{\mathrm{T}} P \eta_{i+1}(t-h) \\
& -2 \eta_{i}^{\mathrm{T}}(t) P \psi_{i},
\end{aligned}
$$

and, for $i=N_{k}$, the time derivative of $W_{i}$ along the trajectory (25) is

$$
\begin{aligned}
\dot{W}_{i}= & \eta_{i}^{\mathrm{T}}(t) P \dot{\eta}_{i}(t) \\
= & \eta_{i}^{\mathrm{T}}(t)\left(A^{\mathrm{T}} P+P A-2 \lambda_{k} P D D^{\mathrm{T}} P\right) \eta_{i}(t)+2 \eta_{i}^{\mathrm{T}}(t) P \Delta A \eta_{i}(t)-2 \lambda_{k} \eta_{i}^{\mathrm{T}}(t) P \Delta B D^{\mathrm{T}} P \eta_{i}(t-h) \\
& -2 \eta_{i}^{\mathrm{T}}(t) P \psi_{i} \\
\leq & \eta_{i}^{\mathrm{T}}(t)\left(A^{\mathrm{T}} P+P A-2 \lambda_{k} P D D^{\mathrm{T}} P\right) \eta_{i}(t)+\frac{1}{\mu} \eta_{i}^{\mathrm{T}}(t) P E E^{\mathrm{T}} P \eta_{i}(t)+\mu \eta_{i}^{\mathrm{T}}(t) F_{1}^{\mathrm{T}} F_{1} \eta_{i}(t) \\
& +\frac{1}{\epsilon} \lambda_{k} \eta_{i}^{\mathrm{T}}(t) P E E^{\mathrm{T}} P \eta_{i}(t)+\epsilon \lambda_{k} \eta_{i}^{\mathrm{T}}(t-h) P D F_{2}^{\mathrm{T}} F_{2} D^{\mathrm{T}} P \eta_{i}(t-h)-2 \eta_{i}^{\mathrm{T}}(t) P \psi_{i} \\
= & \eta_{i}^{\mathrm{T}}(t)\left[A^{\mathrm{T}} P+P A-2 \lambda_{k} P D D^{\mathrm{T}} P+\left(\frac{\lambda_{k}}{\epsilon}+\frac{1}{\mu}\right) P E E^{\mathrm{T}} P+\mu F_{1}^{\mathrm{T}} F_{1}\right] \eta_{i}(t) \\
& +\epsilon \lambda_{k} \eta_{i}^{\mathrm{T}}(t-h) P D F_{2}^{\mathrm{T}} F_{2} D^{\mathrm{T}} P \eta_{i}(t-h)-2 \eta_{i}^{\mathrm{T}}(t) P \psi_{i},
\end{aligned}
$$

where the inequality $\pm a^{\mathrm{T}} b \leq a^{\mathrm{T}} a+b^{\mathrm{T}} b$, for any vectors $a$ and $b$, has been used.

Similarly, for $j=n_{k} / 2$ in $(26)^{\dagger}$, we have in pairs

$$
\begin{aligned}
& \dot{W}_{i_{1}}+\dot{W}_{i_{2}} \\
& \leq \eta_{i_{1}}^{\mathrm{T}}(t)\left[A^{\mathrm{T}} P+P A-2 \alpha_{k} P D D^{\mathrm{T}} P+\left(\frac{\alpha_{k}+\beta_{k}}{\epsilon}+\frac{1}{\mu}\right) P E E^{\mathrm{T}} P+\mu F_{1}^{\mathrm{T}} F_{1}\right] \eta_{i_{1}}(t) \\
& +\eta_{i_{2}}^{\mathrm{T}}(t)\left[A^{\mathrm{T}} P+P A-2 \alpha_{k} P D D^{\mathrm{T}} P+\left(\frac{\alpha_{k}+\beta_{k}}{\epsilon}+\frac{1}{\mu}\right) P E E^{\mathrm{T}} P+\mu F_{1}^{\mathrm{T}} F_{1}\right] \eta_{i_{2}}(t) \\
& +\epsilon\left(\alpha_{k}+\beta_{k}\right) \eta_{i_{1}}^{\mathrm{T}}(t-h) P D F_{2}^{\mathrm{T}} F_{2} D^{\mathrm{T}} P \eta_{i_{1}}(t-h)-2 \eta_{i_{1}}(t)^{\mathrm{T}} P \psi_{i_{1}} \\
& +\epsilon\left(\alpha_{k}+\beta_{k}\right) \eta_{i_{2}}^{\mathrm{T}}(t-h) P D F_{2}^{\mathrm{T}} F_{2} D^{\mathrm{T}} P \eta_{i_{2}}(t-h)-2 \eta_{i_{2}}(t)^{\mathrm{T}} P \psi_{i_{2}},
\end{aligned}
$$

${ }^{\dagger}$ We omit without ambiguity the parameter $j$ in $i_{1}(j)$ and $i_{2}(j)$ for simplicity. 
and, for $j=1,2, \ldots, n_{k} / 2-1$,

$$
\begin{aligned}
& \dot{W}_{i_{1}}+\dot{W}_{i_{2}} \\
& \leq \eta_{i_{1}}^{\mathrm{T}}(t)\left[A^{\mathrm{T}} P+P A-2\left(\alpha_{k}-1\right) P D D^{\mathrm{T}} P+\left(\frac{\alpha_{k}+\beta_{k}+1}{\epsilon}+\frac{1}{\mu}\right) P E E^{\mathrm{T}} P+\mu F_{1}^{\mathrm{T}} F_{1}\right] \eta_{i_{1}}(t) \\
& +\eta_{i_{2}}^{\mathrm{T}}(t)\left[A^{\mathrm{T}} P+P A-2\left(\alpha_{k}-1\right) P D D^{\mathrm{T}} P+\left(\frac{\alpha_{k}+\beta_{k}+1}{\epsilon}+\frac{1}{\mu}\right) P E E^{\mathrm{T}} P+\mu F_{1}^{\mathrm{T}} F_{1}\right] \eta_{i_{2}}(t) \\
& +\epsilon\left(\alpha_{k}+\beta_{k}\right) \eta_{i_{1}}^{\mathrm{T}}(t-h) P D F_{2}^{\mathrm{T}} F_{2} D^{\mathrm{T}} P \eta_{i_{1}}(t-h)+\eta_{i_{1}+2}^{\mathrm{T}}(t) P D D^{\mathrm{T}} P \eta_{i_{1}+2}(t)-2 \eta_{i_{1}}(t)^{\mathrm{T}} P \psi_{i_{1}} \\
& +\epsilon\left(\alpha_{k}+\beta_{k}\right) \eta_{i_{2}}^{\mathrm{T}}(t-h) P D F_{2}^{\mathrm{T}} F_{2} D^{\mathrm{T}} P \eta_{i_{2}}(t-h)+\eta_{i_{2}+2}^{\mathrm{T}}(t) P D D^{\mathrm{T}} P \eta_{i_{2}+2}(t)-2 \eta_{i_{2}}(t)^{\mathrm{T}} P \psi_{i_{2}} \\
& +\epsilon \eta_{i_{1}+2}^{\mathrm{T}}(t-h) P D F_{2}^{\mathrm{T}} F_{2} D^{\mathrm{T}} P \eta_{i_{1}+2}(t-h)+\epsilon \eta_{i_{2}}^{\mathrm{T}}(t-h) P D F_{2}^{\mathrm{T}} F_{2} D^{\mathrm{T}} P \eta_{i_{2}+2}(t-h) .
\end{aligned}
$$

where the inequality $\pm a^{\mathrm{T}} b \leq a^{\mathrm{T}} a+b^{\mathrm{T}} b$, for any vectors $a$ and $b$, has been used again.

The above inequalities will be used in the consensus analysis. However, we note that the extra integral term $\psi_{i}(z)$ in the transformed system dynamic model (20) is expressed as a function of the state $z$. For the consensus analysis within the framework of Lyapunov-Krasovskii functionals, we need to establish a bound of the integral function $-2 \eta_{i}^{\mathrm{T}} P \psi_{i}$ in terms of the transformed state $\eta$. The following lemma establishes a bound for the cross term $-2 \eta_{i}^{\mathrm{T}} P \psi_{i}$ with respect to the transformed state $\eta$.

\section{Lemma 3}

For the integral term $\Psi(z)=\left[\psi_{1}(z), \psi_{2}(z), \ldots, \psi_{N}(z)\right]^{\mathrm{T}}$ in (20), the summation of $-2 \eta_{i}^{\mathrm{T}} P \psi_{i}$ is bounded by

$$
\begin{aligned}
-\sum_{i=1}^{N} 2 \eta_{i}^{\mathrm{T}} P \psi_{i} \leq & \frac{2}{\rho} \sum_{i=2}^{N} \eta_{i}^{\mathrm{T}}(t) P E E^{\mathrm{T}} P \eta_{i}(t) \\
& +\rho \gamma_{0}^{2} \sum_{i=2}^{N} h \int_{0}^{h} \eta_{l}^{\mathrm{T}}(t-\tau) P D D^{\mathrm{T}} e^{A^{\mathrm{T}} \tau} F_{1}^{\mathrm{T}} F_{1} e^{A \tau} D D^{\mathrm{T}} P \eta_{l}(t-\tau) \mathrm{d} \tau
\end{aligned}
$$

where $\gamma_{0}^{2}=2\left\|T^{-1}\right\|_{\mathrm{F}}^{2}\left(1+N\|r\|_{2}^{2}\right)\|Q\|_{\mathrm{F}}^{2}\|T\|_{\mathrm{F}}^{2}$.

Proof

See Appendix A.

With the bound derived in Lemma 3, sufficient conditions can be identified respectively for the cases of the Laplacian matrix with distinct eigenvalues and multiple eigenvalues to guarantee the consensus. The following theorem summarises the results.

\section{Theorem 1}

Consider multi-agent systems (1) with Assumptions 1 and 2. The consensus control problem of system (1) can be solved by the control design (11) with the control gain $K=D^{\mathrm{T}} P$, if there exist matrices $X=P^{-1}>0, Y>0$ and scalars $\mu>0, \epsilon>0, \rho>0$, such that

$$
\begin{aligned}
& {\left[\begin{array}{cc}
Y & D F_{2}^{\mathrm{T}} \\
F_{2} D^{\mathrm{T}} & \frac{1}{\epsilon} I
\end{array}\right]>0} \\
& {\left[\begin{array}{ccc}
U & X F_{1}^{\mathrm{T}} & D D^{T} \\
F_{1} X & -\frac{1}{\mu} I & 0 \\
D D^{\mathrm{T}} & 0 & -\frac{1}{\rho \gamma_{0}^{2}} W
\end{array}\right]<0,}
\end{aligned}
$$

where $U$ is specified in one of the following two cases: 
(i) If the eigenvalues of the Laplacian matrix $L$ are distinct,

$$
U=X A^{\mathrm{T}}+A X-2 \underline{\alpha} D D^{\mathrm{T}}+\left(\frac{1}{\mu}+\frac{\bar{\alpha}+\bar{\beta}}{\epsilon}+\frac{2}{\rho}\right) E E^{\mathrm{T}}+(\bar{\alpha}+\bar{\beta}) Y,
$$

(ii) If the Laplacian matrix $L$ has multiple eigenvalues,

$$
U=X A^{\mathrm{T}}+A X-2(\underline{\alpha}-1) D D^{\mathrm{T}}+\left(\frac{1}{\mu}+\frac{\bar{\alpha}+\bar{\beta}+1}{\epsilon}+\frac{2}{\rho}\right) E E^{\mathrm{T}}+(\bar{\alpha}+\bar{\beta}+1) Y,
$$

and

$$
\begin{aligned}
& \gamma_{0}^{2}=2\left\|T^{-1}\right\|_{\mathrm{F}}^{2}\left(1+N\|r\|_{2}^{2}\right)\|Q\|_{\mathrm{F}}^{2}\|T\|_{\mathrm{F}}^{2}, \\
& W^{-1} \geq h \int_{0}^{h} \mathrm{e}^{A^{\mathrm{T}} s} F_{1}^{\mathrm{T}} F_{1} e^{A s} \mathrm{~d} s, \\
& \bar{\alpha}=\max \left\{\lambda_{2}, \ldots, \lambda_{n_{\lambda}}, \alpha_{1}, \ldots, \alpha_{n_{\nu}}\right\}, \\
& \bar{\beta}=\max \left\{\beta_{1}, \ldots, \beta_{n_{v}}\right\} \\
& \underline{\alpha}=\min \left\{\lambda_{2}, \ldots, \lambda_{n_{\lambda}}, \alpha_{1}, \ldots, \alpha_{n_{\nu}}\right\} .
\end{aligned}
$$

Proof

See Appendix B.

\section{Remark 7}

The conditions shown in (33) to (34) can be checked by standard LMI routines for a set of fixed values $R$ and $W^{-1}$. The iterative methods developed in [30] for single linear system may also be applied here.

\section{Remark 8}

Note from (35) and (36) that a more stringent condition is required for the case of the Laplacian matrix with multiple eigenvalues than the case with only distinct eigenvalues.

\section{Remark 9}

It can be seen from (38) that the matrix $W^{-1}$ explicitly depends on the delay $h$, which implies that large input delays will lead to a difficulty in finding a feasible solution satisfying the conditions (33) and (34) simultaneously. Even if such a feasible solution $P$ exists, a larger input delay results in a smaller $P$ and therefore a smaller control gain $K$, which further implies a more sluggish consensus response.

\section{AN EXAMPLE}

In this section, the scenario under consideration is a connection of six subsystems (i.e., $N=6$ ) in the network as shown in Fig.1. The dynamics of each subsystem are described by (1) with

$$
\begin{aligned}
& A=\left[\begin{array}{cc}
-1 & 1 \\
0 & 0
\end{array}\right], \quad B=\left[\begin{array}{l}
0 \\
1
\end{array}\right], \quad \Sigma(t)=\left[\begin{array}{cc}
\sin (t) & 0 \\
0 & \sin (2 t)
\end{array}\right], \\
& E=\left[\begin{array}{ll}
2 & 0 \\
0 & 2
\end{array}\right], \quad F_{1}=\left[\begin{array}{cc}
0.1 & 0 \\
0 & 0.1
\end{array}\right], F_{2}=\left[\begin{array}{l}
0.1 \\
0.1
\end{array}\right] .
\end{aligned}
$$

The Laplacian matrix associated with the graph in Fig. 1 is

$$
L=\left[\begin{array}{cccccc}
3 & 0 & 0 & -1 & -1 & -1 \\
-1 & 1 & 0 & 0 & 0 & 0 \\
-1 & -1 & 2 & 0 & 0 & 0 \\
-1 & 0 & 0 & 1 & 0 & 0 \\
0 & 0 & 0 & -1 & 1 & 0 \\
0 & 0 & 0 & 0 & -1 & 1
\end{array}\right]
$$




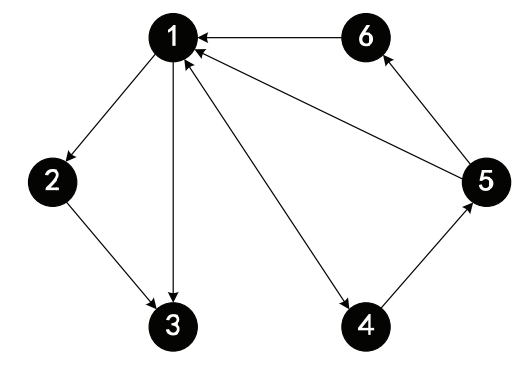

Figure 1. Network Connection Topology

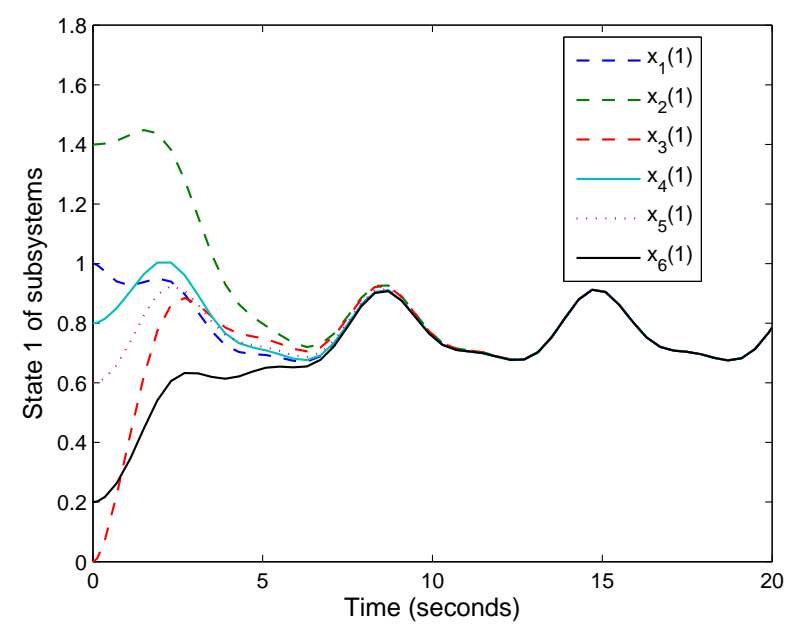

Figure 2. The state 1 of subsystems with $h=0.03$.

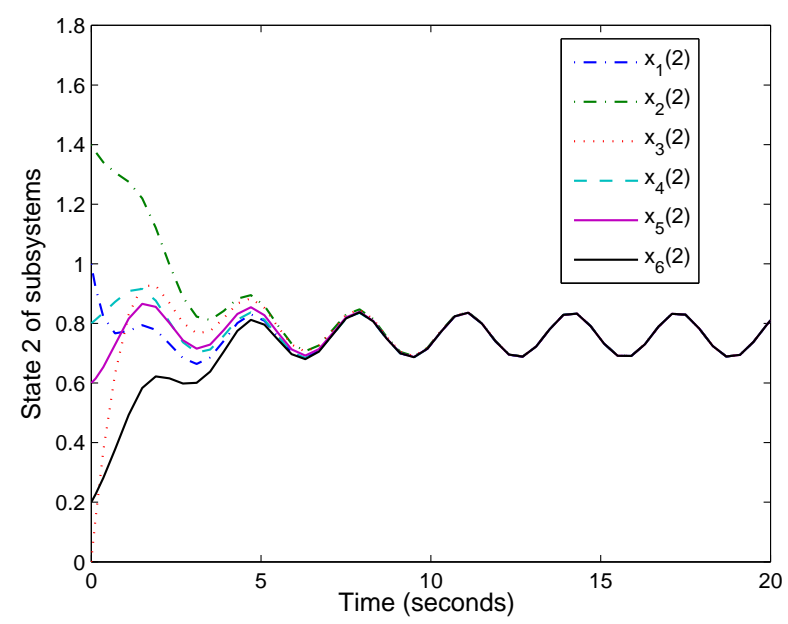

Figure 3 . The state 2 of subsystems with $h=0.03$.

where the eigenvalues of $L$ are $[0,1,2,3.3247,1.3376 \pm j 0.5623]$, which implies the case (i) in Theorem 1 is satisfied. Then, it can be straightforward to calculate the Jordan canonical form of 
$L$ as

$$
J=\left[\begin{array}{cccccc}
0 & 0 & 0 & 0 & 0 & 0 \\
0 & 2 & 0 & 0 & 0 & 0 \\
0 & 0 & 1 & 0 & 0 & 0 \\
0 & 0 & 0 & 3.3247 & 0 & 0 \\
0 & 0 & 0 & 0 & 1.3376 & 0.5623 \\
0 & 0 & 0 & 0 & -0.5623 & 1.3376
\end{array}\right]
$$

with the matrices

$$
\begin{aligned}
& T=\left[\begin{array}{cccccc}
1 & 0 & 0 & -12.5635 & 0.2818-\mathrm{j} 0.0145 & 0.2818-\mathrm{j} 0.0145 \\
1 & 0 & 1 & 5.4043 & -0.2022+\mathrm{j} 0.3797 & -0.2022-\mathrm{j} 0.3797 \\
1 & 1 & 1 & 5.4043 & -0.2022+\mathrm{j} 0.3797 & -0.2022-\mathrm{j} 0.3797 \\
1 & 0 & 0 & 5.4043 & -0.2022+\mathrm{j} 0.3797 & -0.2022-\mathrm{j} 0.3797 \\
1 & 0 & 0 & -2.3247 & -0.3376-\mathrm{j} 0.5623 & -0.3376+\mathrm{j} 0.5623 \\
1 & 0 & 0 & 1 & 1 & 1
\end{array}\right] \\
& T^{-1}=\left[\begin{array}{cccccc}
0.1429 & 0 & 0 & 0.4286 & 0.2857 & 0.1429 \\
0 & -1 & 1 & 0 & 0 & 0 \\
0 & 1.0000 & 0 & -1.0000 & 0 & 0 \\
-0.0705 & 0 & 0 & 0.0229 & 0.0173 & 0.0303 \\
-0.0362-\mathrm{j} 0.2945 & 0 & 0 & -0.2257-\mathrm{j} 0.4693 & -0.1515+\mathrm{j} 0.5799 & 0.4134+\mathrm{j} 0.1839 \\
-0.0362+\mathrm{j} 0.2945 & 0 & 0 & -0.2257+\mathrm{j} 0.4693 & -0.1515-\mathrm{j} 0.5799 & 0.4134-\mathrm{j} 0.1839
\end{array}\right] .
\end{aligned}
$$

Thus, we have $r=[0.1429,0,0,0.4286,0.2857,0.1429]^{\mathrm{T}}, \underline{\alpha}=1, \bar{\alpha}=3.3247$ and $\bar{\beta}=0.5623$.

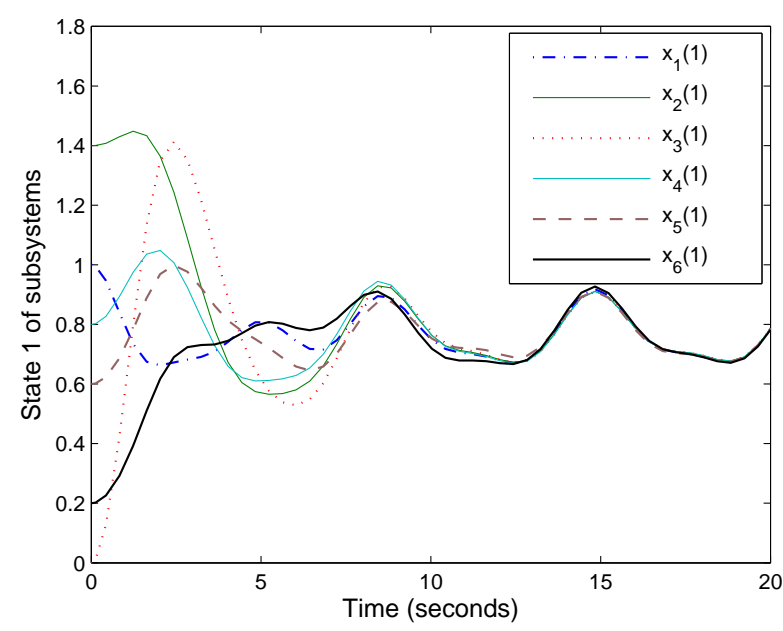

Figure 4 . The state 1 of subsystems with $h=0.3 \mathrm{sec}$.

The input delay of the system is $h=0.03 \mathrm{sec}$. The positive definite matrix $P$ can be computed with $\mu=1, \epsilon=1$ and $\rho=0.1$, as

$$
X^{-1}=P=\left[\begin{array}{ll}
0.0002 & 0.0002 \\
0.0002 & 0.5174
\end{array}\right]
$$

to satisfy the conditions of Theorem 1. Consequently, the control gain is obtained as

$$
K=D^{\mathrm{T}} P=\left[\begin{array}{ll}
0.0002 & 0.5173
\end{array}\right] .
$$

Simulation study has been carried out with the results shown in Figures 2 and 3 for the states of each subsystem. Clearly the conditions specified in Theorem 1 are sufficient for the control gain 


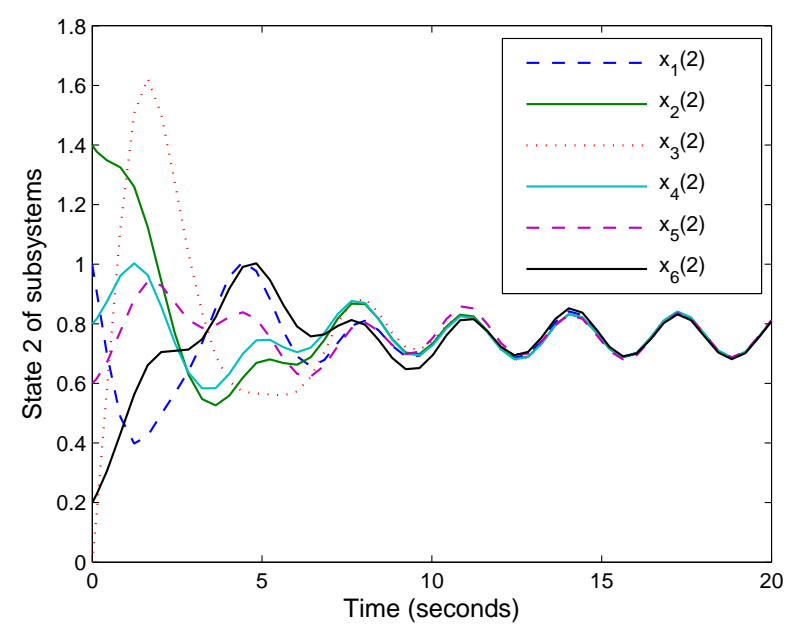

Figure 5. The state 2 of subsystems with $h=0.3 \mathrm{sec}$.

to achieve consensus control. Without any re-tuning the control gain, the consensus control is still achieved for the multi-agent system with much a much larger delay $h=0.3 \mathrm{sec}$, as shown in Figures 4 and 5, which imply the conditions could be conservative in the control gain design for a given input delay.

\section{CONCLUSION}

In this paper, we have solved the consensus problem of multi-agent systems in the presence of parametric uncertainty and input delay by exploiting the reduction method for delay together with consensus control design based on real Jordan form of the Laplacian matrix. Further analysis has been developed to tackle the influence of the extra integral term under transformations due to the model uncertainty. Sufficient conditions are derived for the closed-loop system to achieve global consensus using Lyapunov-Krasovskii method in the time domain. The significance of this research is to provide a feasible method to deal with the robust consensus control for input-delayed uncertain multi-agent systems.

\section{ACKNOWLEDGEMENT}

This work was supported by National Natural Science Foundation (NNSF) of China under Grants 61203022, 61473010 and the China Scholarship Council (CSC).

\section{APPENDIX}

\section{A. Proof of Lemma 3}

From the state transformations (17) and (19), we have

$$
\Psi(z)=\left(T^{-1} \otimes I_{n}\right)\left(M \otimes I_{n}\right)\left(I_{N} \otimes \Delta A\right) \sigma .
$$

Let

$$
\begin{aligned}
\Phi & =\left[\phi_{1}, \ldots, \phi_{N}\right]^{\mathrm{T}}=\left(M \otimes I_{n}\right) \bar{\sigma}, \\
\bar{\sigma} & =\left[\bar{\sigma}_{1}, \ldots, \bar{\sigma}_{N}\right]^{\mathrm{T}}=\left(I_{N} \otimes \Delta A\right) \sigma .
\end{aligned}
$$


Recalling $M=I_{N}-\mathbf{1} r^{\mathrm{T}}$, we have

$$
\begin{aligned}
\phi_{k} & =\bar{\sigma}_{k}-\sum_{j=1}^{N} r_{j} \bar{\sigma}_{j}=\Delta A\left(\sigma_{k}-\sum_{j=1}^{N} r_{j} \sigma_{j}\right) \\
\psi_{i} & =\left(\tau_{i} \otimes I_{n}\right) \Phi=\sum_{k=1}^{N} \tau_{i k} \phi_{k}=\sum_{k=1}^{N} \tau_{i k} \Delta A\left(\sigma_{k}-\sum_{j=1}^{N} r_{j} \sigma_{j}\right) \\
& =\Delta A \sum_{k=1}^{N} \tau_{i k} \sigma_{k}-\Delta A \sum_{k=1}^{N} \tau_{i k} \sum_{j=1}^{N} r_{j} \sigma_{j},
\end{aligned}
$$

where $\tau_{i}$ is the $i$ th row of $T^{-1}$. It then follows that

$$
\begin{aligned}
-2 \eta_{i}^{\mathrm{T}} P \psi_{i}= & 2 \eta_{i}^{\mathrm{T}} P \Delta A \sum_{k=1}^{N} \tau_{i k} \sum_{j=1}^{N} r_{j} \sigma_{j}-2 \eta_{i}^{\mathrm{T}} P \Delta A \sum_{k=1}^{N} \tau_{i k} \sigma_{k} \\
\leq & \frac{2}{\rho} \eta_{i}^{\mathrm{T}} P E E^{\mathrm{T}} P \eta_{i}+\rho\left(\sum_{k=1}^{N} \tau_{i k} \sigma_{k}\right)^{\mathrm{T}} F_{1}^{\mathrm{T}} F_{1}\left(\sum_{k=1}^{N} \tau_{i k} \sigma_{k}\right) \\
& +\rho\left(\sum_{k=1}^{N} \tau_{i k} \sum_{j=1}^{N} r_{j} \sigma_{j}\right)^{\mathrm{T}} F_{1}^{\mathrm{T}} F_{1}\left(\sum_{k=1}^{N} \tau_{i k} \sum_{j=1}^{N} r_{j} \sigma_{j}\right) \\
\leq & \frac{2}{\rho} \eta_{i}^{\mathrm{T}} P E E^{\mathrm{T}} P \eta_{i}+\rho\left(\left\|\tau_{i}\right\|_{2}^{2}+\left\|\tau_{i}\right\|_{1}^{2}\|r\|_{2}^{2}\right) \sum_{k=1}^{N}\left\|F_{1} \sigma_{k}\right\|_{2}^{2} .
\end{aligned}
$$

From (11) and (15), we have

$$
\begin{aligned}
\sigma_{k} & =-\int_{t}^{t+h} \mathrm{e}^{A(t-\tau)} B K \sum_{j=1}^{N} l_{k j} z_{j}(\tau-h) \mathrm{d} \tau \\
& =\int_{t}^{t+h} \mathrm{e}^{A(t-\tau)} B K \sum_{j=1}^{N} q_{k j}\left[z_{j}(\tau-h)-z_{k}(\tau-h)\right] \mathrm{d} \tau \\
& =\int_{t}^{t+h} \mathrm{e}^{A(t-\tau)} B K \sum_{j=1}^{N} q_{k j}\left[\left(t_{j}-t_{k}\right) \otimes I_{n}\right] \eta(\tau-h) \mathrm{d} \tau \\
& =\int_{t}^{t+h} \mathrm{e}^{A(t-\tau)} B K \sum_{j=1}^{N} q_{k j} \sum_{l=1}^{N}\left(t_{j l}-t_{k l}\right) \eta_{l}(\tau-h) \mathrm{d} \tau \\
& =\sum_{j=1}^{N} q_{k j} \sum_{l=1}^{N}\left(t_{j l}-t_{k l}\right) \delta_{l},
\end{aligned}
$$

where $t_{i}$ is the $i$ th row of $T$ and

$$
\delta_{l}=\int_{t}^{t+h} \mathrm{e}^{A(t-\tau)} B K \eta_{l}(\tau-h) \mathrm{d} \tau .
$$


It then follows that

$$
\begin{aligned}
\sum_{k=1}^{N}\left\|F_{1} \sigma_{k}\right\|_{2}^{2} & =\sum_{k=1}^{N}\left\|\sum_{j=1}^{N} q_{k j} \sum_{l=1}^{N}\left(t_{j l}-t_{k l}\right) F_{1} \delta_{l}\right\|_{2}^{2} \\
& \leq \sum_{k=1}^{N}\left\|\sum_{j=1}^{N} q_{k j} \sum_{l=1}^{N} t_{j l} F_{1} \delta_{l}\right\|_{2}^{2}+\sum_{k=1}^{N}\left\|\sum_{j=1}^{N} q_{k j} \sum_{l=1}^{N} t_{k l} F_{1} \delta_{l}\right\|_{2}^{2} \\
& \leq 2\|Q\|_{\mathrm{F}}^{2}\|T\|_{\mathrm{F}}^{2} \sum_{l=1}^{N}\left\|F_{1} \delta_{l}\right\|_{2}^{2} .
\end{aligned}
$$

We next deal with $\left\|F_{1} \delta_{l}\right\|_{2}^{2}$ in (45). Then, using Lemma 2 we have

$$
\begin{aligned}
\left\|F_{1} \delta_{l}\right\|_{2}^{2} & =\delta_{l}^{\mathrm{T}} F_{1}^{\mathrm{T}} F_{1} \delta_{l} \\
& =\left(\int_{t}^{t+h} F_{1} \mathrm{e}^{A(t-\tau)} B K \eta_{l}(\tau-h) \mathrm{d} \tau\right)^{\mathrm{T}}\left(\int_{t}^{t+h} F_{1} \mathrm{e}^{A(t-\tau)} B K \eta_{l}(\tau-h) \mathrm{d} \tau\right) \\
& \leq h \int_{t}^{t+h} \eta_{l}^{\mathrm{T}}(\tau-h) K^{\mathrm{T}} D^{\mathrm{T}} e^{A^{\mathrm{T}}(t-\tau+h)} F_{1}^{\mathrm{T}} F_{1} e^{A(t-\tau+h)} D K \eta_{l}(\tau-h) \mathrm{d} \tau \\
& =h \int_{0}^{h} \eta_{l}^{\mathrm{T}}(t-\tau) P D D^{\mathrm{T}} e^{A^{\mathrm{T}} \tau} F_{1}^{\mathrm{T}} F_{1} e^{A \tau} D D^{\mathrm{T}} P \eta_{l}(t-\tau) \mathrm{d} \tau .
\end{aligned}
$$

With (45)-(46) and $\eta_{1} \equiv 0$, the summation of $-2 \eta_{i}^{T} P \psi_{i}$ can be obtained as

$$
\begin{aligned}
-\sum_{i=2}^{N} 2 \eta_{i}^{\mathrm{T}} P \psi_{i} \leq & \frac{2}{\rho} \sum_{i=2}^{N} \eta_{i}^{\mathrm{T}}(t) P E E^{\mathrm{T}} P \eta_{i}(t)+2 \rho \sum_{i=2}^{N}\left(\left\|\tau_{i}\right\|_{2}^{2}+\left\|\tau_{i}\right\|_{1}^{2}\|r\|_{2}^{2}\right)\|Q\|_{\mathrm{F}}^{2}\|T\|_{\mathrm{F}}^{2} \sum_{l=2}^{N}\left\|F_{1} \delta_{l}\right\|_{2}^{2} \\
\leq & \frac{2}{\rho} \sum_{i=2}^{N} \eta_{i}^{\mathrm{T}}(t) P E E^{\mathrm{T}} P \eta_{i}(t) \\
& +\rho \gamma_{0}^{2} \sum_{i=2}^{N} h \int_{0}^{h} \eta_{l}^{\mathrm{T}}(t-\tau) P D D^{\mathrm{T}} e^{A^{\mathrm{T}} \tau} F_{1}^{\mathrm{T}} F_{1} e^{A \tau} D D^{\mathrm{T}} P \eta_{l}(t-\tau) \mathrm{d} \tau
\end{aligned}
$$

where $2 \sum_{i=2}^{N}\left(\left\|\tau_{i}\right\|_{2}^{2}+\left\|\tau_{i}\right\|_{1}^{2}\|r\|_{2}^{2}\right)\|Q\|_{\mathrm{F}}^{2}\|T\|_{\mathrm{F}}^{2} \leq 2\left\|T^{-1}\right\|_{\mathrm{F}}^{2}\left(1+N\|r\|_{2}^{2}\right)\|Q\|_{\mathrm{F}}^{2}\|T\|_{\mathrm{F}}^{2}$ has been inserted in the last inequality with $\sum_{i=1}^{N}\left\|\tau_{i}\right\|_{2}^{2}=\left\|T^{-1}\right\|_{\mathrm{F}}^{2}$ being used.

\section{B. Proof of Theorem 1}

For all the state variables associate with the Jordan blocks of real eigenvalues, we consider the following summation of (27):

$$
V_{k}=\sum_{j=1}^{n_{k}} W_{j+N_{k-1}}
$$

and from (28)-(29) we then obtain

$$
\begin{aligned}
\dot{V}_{k} \leq & \sum_{j=1}^{n_{k}} \eta_{j+N_{k-1}}^{\mathrm{T}}(t)\left[A^{\mathrm{T}} P+P A-2\left(\lambda_{k}-1\right) P D D^{\mathrm{T}} P+\left(\frac{\lambda_{k}+1}{\epsilon}+\frac{1}{\mu}\right) P E E^{\mathrm{T}} P+\mu F_{1}^{\mathrm{T}} F_{1}\right] \\
& \times \eta_{j+N_{k-1}}(t)-\eta_{1+N_{k-1}}^{\mathrm{T}}(t) P D D^{\mathrm{T}} P \eta_{1+N_{k-1}}(t)-\eta_{N_{k}}^{\mathrm{T}}(t) P D D^{\mathrm{T}} P \eta_{N_{k}}(t) \\
& -\frac{1}{\epsilon} \eta_{N_{k}}^{\mathrm{T}}(t) P E E^{\mathrm{T}} P \eta_{N_{k}}(t)+\sum_{j=1}^{n_{k}} \epsilon\left(1+\lambda_{k}\right) \eta_{j+N_{k-1}}^{\mathrm{T}}(t-h) P D F_{2}^{\mathrm{T}} F_{2} D^{\mathrm{T}} P \eta_{j+N_{k-1}}(t-h) \\
& -\epsilon \eta_{1+N_{k-1}}^{\mathrm{T}}(t-h) P D F_{2}^{\mathrm{T}} F_{2} D^{\mathrm{T}} P \eta_{1+N_{k-1}}(t-h)-2 \sum_{j=1}^{n_{k}} \eta_{j+N_{k-1}}^{\mathrm{T}}(t) P \psi_{i} .
\end{aligned}
$$


For all the state variables corresponding to the conjugate eigenvalues in the Jordan blocks, we consider the following summation of (27) in pairs:

$$
V_{k}=\sum_{j=1}^{n_{k} / 2}\left[W_{i_{1}(j)}+W_{i_{2}(j)}\right]
$$

By (30)-(31) and after simple mechanical calculation, we have

$$
\begin{aligned}
& \dot{V}_{k}= \sum_{j=1}^{n_{k} / 2}\left[\dot{W}_{i_{1}(j)}+\dot{W}_{i_{2}(j)}\right] \\
& \leq \sum_{j=1}^{n_{k} / 2} \eta_{2 j-1+N_{k-1}}^{\mathrm{T}}(t)\left[A^{\mathrm{T}} P+P A-2\left(\lambda_{k}-1\right) P D D^{\mathrm{T}} P+\left(\frac{\alpha_{k}+\beta_{k}+1}{\epsilon}+\frac{1}{\mu}\right) P E E^{\mathrm{T}} P+\mu F_{1}^{\mathrm{T}} F_{1}\right] \\
& \times \eta_{2 j-1+N_{k-1}}(t)-\sum_{j=1}^{n_{k} / 2} 2 \eta_{2 j-1+N_{k-1}}^{\mathrm{T}}(t) P \psi_{2 j-1+N_{k-1}} \\
&+\sum_{j=1}^{n_{k} / 2} \eta_{2 j+N_{k-1}}^{\mathrm{T}}(t)\left[A^{\mathrm{T}} P+P A-2\left(\lambda_{k}-1\right) P D D^{\mathrm{T}} P+\left(\frac{\alpha_{k}+\beta_{k}+1}{\epsilon}+\frac{1}{\mu}\right) P E E^{\mathrm{T}} P+\mu F_{1}^{\mathrm{T}} F_{1}\right] \\
& \times \eta_{2 j+N_{k-1}}(t)-\sum_{j=1}^{n_{k} / 2} 2 \eta_{2 j+N_{k-1}}^{\mathrm{T}}(t) P \psi_{2 j+N_{k-1}} \\
&-\eta_{1+N_{k-1}}^{\mathrm{T}}(t) P D D^{\mathrm{T}} P \eta_{1+N_{k-1}}(t)-\eta_{2+N_{k-1}}^{\mathrm{T}}(t) P D D^{\mathrm{T}} P \eta_{2+N_{k-1}}(t)-\eta_{N_{k}-1}^{\mathrm{T}}(t) P D D^{\mathrm{T}} P \eta_{N_{k}-1}(t) \\
&-\eta_{N_{k}}^{\mathrm{T}}(t) P D D^{\mathrm{T}} P \eta_{N_{k}}(t)-\frac{1}{\epsilon} \eta_{N_{k}-1}^{\mathrm{T}}(t) P E E^{\mathrm{T}} P \eta_{N_{k}-1}(t)-\frac{1}{\epsilon} \eta_{N_{k}}^{\mathrm{T}}(t) P E E^{\mathrm{T}} P \eta_{N_{k}}(t) \\
&+\sum_{k} / 2 \\
&+\sum_{j=1} \epsilon\left(\alpha_{k}+\beta_{k}+1\right) \eta_{2 j-1+N_{k-1}}^{\mathrm{T}}(t-h) P D F_{2}^{\mathrm{T}} F_{2} D^{\mathrm{T}} P \eta_{2 j-1+N_{k-1}}(t-h) \\
&+\sum_{j=1}^{n_{k} / 2} \epsilon\left(\alpha_{k}+\beta_{k}+1\right) \eta_{2 j+N_{k-1}}^{\mathrm{T}}(t-h) P D F_{2}^{\mathrm{T}} F_{2} D^{\mathrm{T}} P \eta_{2 j+N_{k-1}}(t-h) \\
&-\epsilon \eta_{N_{k}-1}^{\mathrm{T}}(t-h) P D F_{2}^{\mathrm{T}} F_{2} D^{\mathrm{T}} P \eta_{N_{k}-1}(t-h)-\epsilon \eta_{N_{k}}^{\mathrm{T}}(t-h) P D F_{2}^{\mathrm{T}} F_{2} D^{\mathrm{T}} P \eta_{N_{k}}(t-h) . \\
&(50)
\end{aligned}
$$

With (47) and (49), we consider the following Lyapunov function

$$
\begin{aligned}
\mathcal{V}_{1} & =\sum_{k=2}^{q} V_{k}=\sum_{k=2}^{p} V_{k}+\sum_{k=p+1}^{q} V_{k} \\
& =\sum_{k=2}^{p} \sum_{j=1}^{n_{k}} W_{j+N_{k-1}}+\sum_{k=p+1}^{q} \sum_{j=1}^{n_{k} / 2}\left[W_{i_{1}(j)}+W_{i_{2}(j)}\right],
\end{aligned}
$$


where $N_{k}$ is defined in (23) with $N_{1}=n_{1}=1$ and $2 \leq k \leq q, i_{1}(j)$ and $i_{2}(j)$ are defined in (26) and (??), respectively. By (48) and (50), we can compute the time derivative of (51) as

$$
\begin{aligned}
& \dot{\mathcal{V}}_{1} \leq \sum_{k=2}^{p} \sum_{j=1}^{n_{k}} \eta_{j+N_{k-1}}^{\mathrm{T}}(t)\left[A^{\mathrm{T}} P+P A-2\left(\lambda_{k}-1\right) P D D^{\mathrm{T}} P+\left(\frac{\lambda_{k}+1}{\epsilon}+\frac{1}{\mu}\right) P E E^{\mathrm{T}} P+\mu F_{1}^{\mathrm{T}} F_{1}\right] \\
& \times \eta_{j+N_{k-1}}(t) \\
& +\sum_{k=p+1}^{q} \sum_{j=1}^{n_{k} / 2} \eta_{2 j-1+N_{k-1}}^{\mathrm{T}}(t)\left[A^{\mathrm{T}} P+P A-2\left(\lambda_{k}-1\right) P D D^{\mathrm{T}} P+\left(\frac{\alpha_{k}+\beta_{k}+1}{\epsilon}\right.\right. \\
& \left.\left.+\frac{1}{\mu}\right) P E E^{\mathrm{T}} P+\mu F_{1}^{\mathrm{T}} F_{1}\right] \eta_{2 j-1+N_{k-1}}(t) \\
& +\sum_{k=p+1}^{q} \sum_{j=1}^{n_{k} / 2} \eta_{2 j+N_{k-1}}^{\mathrm{T}}(t)\left[A^{\mathrm{T}} P+P A-2\left(\lambda_{k}-1\right) P D D^{\mathrm{T}} P+\left(\frac{\alpha_{k}+\beta_{k}+1}{\epsilon}\right.\right. \\
& \left.\left.+\frac{1}{\mu}\right) P E E^{\mathrm{T}} P+\mu F_{1}^{\mathrm{T}} F_{1}\right] \eta_{2 j+N_{k-1}}(t) \\
& -\sum_{k=2}^{p} \underbrace{\left[\eta_{1+N_{k-1}}^{\mathrm{T}}(t) P D D^{\mathrm{T}} P \eta_{1+N_{k-1}}(t)+\eta_{N_{k}}^{\mathrm{T}}(t) P D D^{\mathrm{T}} P \eta_{N_{k}}(t)\right]}_{\geq 0} \\
& -\sum_{k=p+1}^{q} \underbrace{\left[\eta_{1+N_{k-1}}^{\mathrm{T}}(t) P D D^{\mathrm{T}} P \eta_{1+N_{k-1}}(t)+\eta_{2+N_{k-1}}^{\mathrm{T}}(t) P D D^{\mathrm{T}} P \eta_{2+N_{k-1}}(t)\right]}_{\geq 0} \\
& -\sum_{k=p+1}^{q} \underbrace{\left[\eta_{N_{k}-1}^{\mathrm{T}}(t) P D D^{\mathrm{T}} P \eta_{N_{k}-1}(t)+\eta_{N_{k}}^{\mathrm{T}}(t) P D D^{\mathrm{T}} P \eta_{N_{k}}(t)\right]}_{\geq 0} \\
& -\sum_{k=2}^{p} \frac{1}{\epsilon} \underbrace{\eta_{N_{k}}^{\mathrm{T}}(t) P E E^{\mathrm{T}} P \eta_{N_{k}}(t)}_{\geq 0}-\sum_{k=p+1}^{q} \frac{1}{\epsilon} \underbrace{\left[\eta_{N_{k}-1}^{\mathrm{T}}(t) P E E^{\mathrm{T}} P \eta_{N_{k}-1}(t)+\eta_{N_{k}}^{\mathrm{T}}(t) P E E^{\mathrm{T}} P \eta_{N_{k}}(t)\right]}_{\geq 0} \\
& +\sum_{k=2}^{p} \sum_{j=1}^{n_{k}} \epsilon\left(1+\lambda_{k}\right) \eta_{j+N_{k-1}}^{\mathrm{T}}(t-h) P D F_{2}^{\mathrm{T}} F_{2} D^{\mathrm{T}} P \eta_{j+N_{k-1}}(t-h) \\
& +\sum_{k=p+1}^{q} \sum_{j=1}^{n_{k} / 2} \epsilon\left(\alpha_{k}+\beta_{k}+1\right) \eta_{2 j-1+N_{k-1}}^{\mathrm{T}}(t-h) P D F_{2}^{\mathrm{T}} F_{2} D^{\mathrm{T}} P \eta_{2 j-1+N_{k-1}}(t-h) \\
& +\sum_{k=p+1}^{q} \sum_{j=1}^{n_{k} / 2} \epsilon\left(\alpha_{k}+\beta_{k}+1\right) \eta_{2 j+N_{k-1}}^{\mathrm{T}}(t-h) P D F_{2}^{\mathrm{T}} F_{2} D^{\mathrm{T}} P \eta_{2 j+N_{k-1}}(t-h) \\
& -\sum_{k=2}^{p} \epsilon \underbrace{\eta_{1+N_{k-1}}^{\mathrm{T}}(t-h) P D F_{2}^{\mathrm{T}} F_{2} D^{\mathrm{T}} P \eta_{1+N_{k-1}}(t-h)}_{\geq 0} \\
& -\sum_{k=p+1}^{q} \epsilon \underbrace{\left[\eta_{N_{k}-1}^{\mathrm{T}}(t-h) P D F_{2}^{\mathrm{T}} F_{2} D^{\mathrm{T}} P \eta_{N_{k}-1}(t-h)+\eta_{N_{k}}^{\mathrm{T}}(t-h) P D F_{2}^{\mathrm{T}} F_{2} D^{\mathrm{T}} P \eta_{N_{k}}(t-h)\right]}_{\geq 0} \\
& -2 \sum_{i=2}^{N} \eta_{i}^{\mathrm{T}}(t) P \psi_{i}(z)
\end{aligned}
$$

Substituting (32) into (52) and using the definitions (39)-(41), we have the following two cases: 
(i) when the Laplacian matrix $L$ has distinct eigenvalues, i.e., $n_{k}=1$ for all $k \in\{2,3, \ldots, q\}$,

$$
\begin{aligned}
\dot{\mathcal{V}}_{1} \leq & \sum_{i=2}^{N} \eta_{i}^{\mathrm{T}}(t)\left[A^{\mathrm{T}} P+P A-2 \underline{\alpha} P D D^{\mathrm{T}} P+\left(\frac{1}{\mu}+\frac{\bar{\alpha}+\bar{\beta}}{\epsilon}+\frac{2}{\rho}\right) P E E^{\mathrm{T}} P+\mu F_{1}^{\mathrm{T}} F_{1}\right] \eta_{i}(t) \\
& +\sum_{i=2}^{N} \epsilon(\bar{\alpha}+\bar{\beta}) \eta_{i}^{\mathrm{T}}(t-h) P D F_{2}^{\mathrm{T}} F_{2} D^{\mathrm{T}} P \eta_{i}(t-h) \\
& +\rho \gamma_{0}^{2} \sum_{i=2}^{N} h \int_{0}^{h} \eta_{i}^{\mathrm{T}}(t-\tau) P D D^{\mathrm{T}} e^{A^{\mathrm{T}} \tau} F_{1}^{\mathrm{T}} F_{1} e^{A \tau} D D^{\mathrm{T}} P \eta_{i}(t-\tau) \mathrm{d} \tau ;
\end{aligned}
$$

(ii) when the Laplacian matrix $L$ has multiple eigenvalues, i.e., $n_{k}>1$ for any $k \in\{2,3, \ldots, q\}$,

$$
\begin{aligned}
\dot{\mathcal{V}}_{1} \leq & \sum_{i=2}^{N} \eta_{i}^{\mathrm{T}}(t)\left[A^{\mathrm{T}} P+P A-2 \underline{\alpha} P D D^{\mathrm{T}} P+\left(\frac{1}{\mu}+\frac{\bar{\alpha}+\bar{\beta}+1}{\epsilon}+\frac{2}{\rho}\right) P E E^{\mathrm{T}} P+\mu F_{1}^{\mathrm{T}} F_{1}\right] \eta_{i}(t) \\
& +\sum_{i=2}^{N} \epsilon(\bar{\alpha}+\bar{\beta}+1) \eta_{i}^{\mathrm{T}}(t-h) P D F_{2}^{\mathrm{T}} F_{2} D^{\mathrm{T}} P \eta_{i}(t-h) \\
& +\rho \gamma_{0}^{2} \sum_{i=2}^{N} h \int_{0}^{h} \eta_{i}^{\mathrm{T}}(t-\tau) P D D^{\mathrm{T}} e^{A^{\mathrm{T}} \tau} F_{1}^{\mathrm{T}} F_{1} e^{A \tau} D D^{\mathrm{T}} P \eta_{i}(t-\tau) \mathrm{d} \tau .
\end{aligned}
$$

For the delayed term shown in (53) and (54), we consider the following Krasovskii functionals for both cases, respectively,

$$
\begin{aligned}
\text { (i) } \mathcal{V}_{2} & =\sum_{i=2}^{N}(\bar{\alpha}+\bar{\beta}) \int_{t-h}^{t} \eta_{i}^{\mathrm{T}}(\tau) R \eta_{i}(\tau) \mathrm{d} \tau, \\
\text { (ii) } \mathcal{V}_{2} & =\sum_{i=2}^{N}(\bar{\alpha}+\bar{\beta}+1) \int_{t-h}^{t} \eta_{i}^{\mathrm{T}}(\tau) R \eta_{i}(\tau) \mathrm{d} \tau .
\end{aligned}
$$

where

$$
R-\epsilon P D F_{2}^{\mathrm{T}} F_{2} D^{\mathrm{T}} P>0 .
$$

A direct calculation gives, respectively, that

$$
\begin{aligned}
\text { (i) } \dot{\mathcal{V}}_{2} & =\sum_{i=2}^{N}(\bar{\alpha}+\bar{\beta})\left[\eta_{i}^{\mathrm{T}}(t) R \eta_{i}(t)-\eta_{i}^{\mathrm{T}}(t-h) R \eta_{i}(t-h)\right], \\
\text { (ii) } \quad \dot{\mathcal{V}}_{2} & =\sum_{i=2}^{N}(\bar{\alpha}+\bar{\beta}+1)\left[\eta_{i}^{\mathrm{T}}(t) R \eta_{i}(t)-\eta_{i}^{\mathrm{T}}(t-h) R \eta_{i}(t-h)\right] .
\end{aligned}
$$

For the integral term shown in (53) or (54), we consider the following Krasovskii functional

$$
\mathcal{V}_{3}=\rho h \gamma_{0}^{2} \sum_{i=2}^{N} \int_{0}^{h} \int_{t-s}^{t} \eta_{i}^{\mathrm{T}}(\tau) P D D^{\mathrm{T}} \mathrm{e}^{A^{\mathrm{T}} s} F_{1}^{\mathrm{T}} F_{1} \mathrm{e}^{A s} D D^{\mathrm{T}} P \eta_{i}(\tau) \mathrm{d} \tau \mathrm{d} s .
$$


A direct calculation gives that

$$
\begin{aligned}
\dot{V}_{3}= & \rho h \gamma_{0}^{2} \sum_{i=2}^{N} \int_{0}^{h} \eta_{i}^{\mathrm{T}}(t) P D D^{\mathrm{T}} \mathrm{e}^{A^{\mathrm{T}} s} F_{1}^{\mathrm{T}} F_{1} \mathrm{e}^{A s} D D^{\mathrm{T}} P \eta_{i}(t) \mathrm{d} s \\
& -\rho h \gamma_{0}^{2} \sum_{i=2}^{N} \int_{0}^{h} \eta_{i}^{\mathrm{T}}(t-s) P D D^{\mathrm{T}} \mathrm{e}^{A^{\mathrm{T}} s} F_{1}^{\mathrm{T}} F_{1} \mathrm{e}^{A s} D D^{\mathrm{T}} P \eta_{i}(t-s) \mathrm{d} s \\
\leq & \rho \gamma_{0}^{2} \sum_{i=2}^{N} \eta_{i}^{\mathrm{T}}(t) P D D^{\mathrm{T}} W^{-1} D D^{\mathrm{T}} P \eta_{i}(t) \\
& -\rho h \gamma_{0}^{2} \sum_{i=2}^{N} \int_{0}^{h} \eta_{i}^{\mathrm{T}}(t-s) P D D^{\mathrm{T}} \mathrm{e}^{A^{\mathrm{T}} s} F_{1}^{\mathrm{T}} F_{1} \mathrm{e}^{A s} D D^{\mathrm{T}} P \eta_{i}(t-s) \mathrm{d} s
\end{aligned}
$$

where

$$
W^{-1} \geq h \int_{0}^{h} \mathrm{e}^{A^{\mathrm{T}} s} F_{1}^{\mathrm{T}} F_{1} \mathrm{e}^{A s} \mathrm{~d} s
$$

Let

$$
\mathcal{V}_{0}=\mathcal{V}_{1}+\mathcal{V}_{2}+\mathcal{V}_{3}
$$

From (53), (57) and (58), we obtain

$$
\dot{\mathcal{V}}_{0}=\dot{\mathcal{V}}_{1}+\dot{\mathcal{V}}_{2}+\dot{\mathcal{V}}_{3} \leq \sum_{i=2}^{N} \eta_{i}^{\mathrm{T}}(t) H \eta_{i}(t)
$$

where

$$
\begin{aligned}
& \text { (i) } \quad H \triangleq A^{\mathrm{T}} P+P A-2 \underline{\alpha} P D D^{\mathrm{T}} P+\mu F_{1}^{\mathrm{T}} F_{1}+(\bar{\alpha}+\bar{\beta}) R \\
& +\left(\frac{1}{\mu}+\frac{\bar{\alpha}+\bar{\beta}}{\epsilon}+\frac{2}{\rho}\right) P E E^{\mathrm{T}} P+\rho \gamma_{0}^{2} P D D^{\mathrm{T}} W^{-1} D D^{\mathrm{T}} P, \\
& \text { or (ii) } \quad H \triangleq A^{\mathrm{T}} P+P A-2(\underline{\alpha}-1) P D D^{\mathrm{T}} P+\mu F_{1}^{\mathrm{T}} F_{1}+(\bar{\alpha}+\bar{\beta}+1) R \\
& +\left(\frac{1}{\mu}+\frac{\bar{\alpha}+\bar{\beta}+1}{\epsilon}+\frac{2}{\rho}\right) P E E^{\mathrm{T}} P+\rho \gamma_{0}^{2} P D D^{\mathrm{T}} W^{-1} D D^{\mathrm{T}} P .
\end{aligned}
$$

From the analysis above, the control (11) stabilises $\eta(t)$ if the conditions (55), (59) and $H<0$ in (60) are satisfied. Indeed, it is easy to see that the conditions (55) and (59) are equivalent, respectively, to the conditions specified in (33) and (38) with $Y=P^{-1} R P^{-1}$. From (60), it can be shown that $H<0$ is equivalent to

$$
\begin{array}{cc}
\text { (i) } P^{-1} A^{T}+A P^{-1}-2 \underline{\alpha} D D^{\mathrm{T}}+\left(\frac{1}{\mu}+\frac{\bar{\alpha}+\bar{\beta}}{\epsilon}+\frac{2}{\rho}\right) E E^{\mathrm{T}} \\
\quad+\mu P^{-1} F_{1}^{\mathrm{T}} F_{1} P^{-1}+(\bar{\alpha}+\bar{\beta}) P^{-1} R P^{-1}+\rho \gamma_{0}^{2} D D^{\mathrm{T}} W^{-1} D D^{\mathrm{T}}<0 \\
\text { or (ii) } \quad P^{-1} A^{\mathrm{T}}+A P^{-1}-2(\underline{\alpha}-1) D D^{\mathrm{T}}+\left(\frac{1}{\mu}+\frac{\bar{\alpha}+\bar{\beta}+1}{\epsilon}+\frac{2}{\rho}\right) E E^{T} \\
\\
\quad+\mu P^{-1} F_{1}^{\mathrm{T}} F_{1} P^{-1}+(\bar{\alpha}+\bar{\beta}+1) P^{-1} R P^{-1}+\rho \gamma_{0}^{2} D D^{\mathrm{T}} W^{-1} D D^{\mathrm{T}}<0
\end{array}
$$

which is further equivalent to (34) with $X=P^{-1}$. Hence, we conclude that $\eta(t)$ converges to zero asymptotically. This completes the proof.

\section{REFERENCES}

1. Dong R, Geng Z. Consensus for formation control of multi-agent systems. International Journal of Robust and Nonlinear Control 2015; 25(14): 2481-2501. 
2. Olfati-Saber R. Flocking for multi-agent dynamic systems: Algorithms and theory. IEEE Transactions on Automatic Control 2006; 51(3): 401-420.

3. Das A, Lewis F. Cooperative adaptive control for synchronization of second-order systems with unknown nonlinearities. International Journal of Robust and Nonlinear Control 2011; 21(13): 1509-1524.

4. Ren W. Information consensus in multivehicle cooperative control. IEEE Control Systems Magazine 2007; 27(2): $71-82$.

5. Olfati-Saber R, Fax J, Murray R. Consensus and cooperation in networked multi-agent systems. Proceedings of the IEEE 2007; 95(1): 215-233.

6. Li Z, Duan Z, Chen G, Huang L. Consensus of multi-agent systems and synchronization of complex networks: a unified viewpoint. IEEE Transactions on Circuits and System I: Regular Papers 2010; 57(1): 213-224.

7. Ding Z. Consensus output regulation of a class of heterogeneous nonlinear systems. IEEE Transactions on Automatic Control 2013; 58(10): 2648-2653.

8. Zuo Z, Tie L. Distributed robust finite-time nonlinear consensus protocols for multi-agent systems. International Journal of Systems Science 2016; 47(6): 1366-1375.

9. Zuo Z, "Nonsingular fixed-time consensus tracking for second-order multi-agent networks," Automatica, 2015; 54: 305-309.

10. Olfati-Saber R, Murray R. Consensus problems in networks of agents with switching topology and time-delay. IEEE Transactions on Automatic Control 2004; 49(9): 1520-1533.

11. Richard J. Time-delay systems: An overview of some recent advances and open problems. Automatica 2003; 39(10): $1667-1694$.

12. Moreau L. Stability of multi-agent systems with time-dependent communication links. IEEE Transactions on Automatic Control 2005; 50(2): 169-182.

13. Meng Z, Ren W, Cao Y, You Z, Leaderless and leader-following consensus with communication and input delays under a directed network topology. IEEE Transactions on Systems, Man, and Cybernetics, Part B: Cybernetics 2011; 41(1): 75-88.

14. Chen G, Lewis F. Robust consensus of multiple inertial agents with coupling delays and variable topologies. International Journal of Robust and Nonlinear Control 2011; 21(6): 666-685.

15. Sheng J, Ding Z. Optimal consensus control of linear multi-agent systems with communication time delay. IET Control Theory and Application 2013; 7(15): 1899-1905.

16. Sun Y, Wang L. Consensus of multi-agent systems in directed networks with non-uniform time-varying delays. IEEE Transactions on Automatic Control 2009; 54(7): 1607-1613.

17. Zhou B, Lin Z. Consensus of high-order multi-agent systems with large input and communication delays. Automatica 2014; 50(2): 452-464.

18. Parlakçı M. Improved robust stability criteria and design of robust stabilizing controller for uncertain linear time delay systems. International Journal of Robust and Nonlinear Control 2006; 16(13): 599-636.

19. Han D, Chesi G, Hung Y. Robust consensus for a class of uncertain multi-agent dynamical systems. IEEE Transactions on Industrial Informatics 2013; 9(1): 306-312.

20. Han D, Chesi G. Robust consensus for uncertain multi-agent systems with discrete-time dynamics. International Journal of Robust and Nonlinear Control 2014; 24(13): 1858-1872.

21. Wang J, Duan Z, Wen G, Chen G. Distributed robust control of uncertain linear multi-gent systems. International Journal of Robust and Nonlinear Control 2015; 25(13): 2162-2179.

22. Tian Y, Liu C. Robust consensus of multi-agent systems with diverse input delays and asymmetric interconnection perturbations. Automatica 2009; 45(5): 1347-1353.

23. Wang P, Jia Y, Du J, Zhang J. Robust $H_{\infty}$ consensus control of uncertain multi-agent systems with nonlinear dynamics and time-varying delays. Proceeding of 2014 American Control Conference, Portland, Oregon, USA, 2014; 2587-2592.

24. Lin P, Jia Y. Robust $H_{\infty}$ consensus analysis of a class of second-order multi-agent systems with uncertainty. IET Control Theory and Application 2010; 4(3): 487-498.

25. Kwon W, Pearson A. Feedback stabilization of linear systems with delayed control. IEEE Transactions on Automatic Control 1980; 25(2): 266-269.

26. Artstein Z. Linear systems with delayed controls: a reduction. IEEE Transactions on Automatic Control 1982; 27(4): 869-879.

27. Jia Y. Robust $H_{\infty}$ control. Beijing: Science Press, 2007. (in Chinese)

28. Ding Z. Consensus control of a class of Lipschitz nonlinear systems. International Journal of Control 2014; 87(11): $2372-2382$.

29. Ding Z, Lin Z. Truncated state prediction for control of Lipschitz nonlinear systems with input delay. Proceedings of the 53rd IEEE Conference on Decision and Control, Los Angeles, CA, USA, 2014; 1966-1971.

30. Yoon S, Lin Z. Truncated predictor feedback control for exponentially unstable linear systems with time-varying input delay. Systems \& Control Letters 2013; 62(10): 837-844.

31. Manitius A. and Olbrot A. Finite spectrum assignment problem for systems with delays. IEEE Transactions on Automatic Control 1979; 24(4): 541-553.

32. Poznyak A., Polyakov A., Azhmyakov V. Attractive Ellipsoids in Robust Control, Birkhäuser: Springer, 2014. 